\title{
Análisis de incertidumbre en calibración de medidores de presión según DKD-R 6-1. Cinco métodos de cálculo
}

\author{
Uncertainty analysis on pressure gauges calibrations \\ according to DKD-R 6-1. Five calculation methods
}

Constantino, Pablo (1), Camacho, Javier (2), Acquarone, Alejandro (1).

(1)Departamento de Metrología, Laboratorio Tecnológico del Uruguay, LATU - (2)VIA University College, Denmark.

Contacto: pconstan@latu.org.uy

RECIBIDO: 23/04/2014 - APROBADO: 30/09/2014

\begin{abstract}
Resumen
Este trabajo presenta un estudio de las contribuciones de incertidumbre e incertidumbre expandida que tienen lugar en la calibración de un medidor digital de presión por comparación directa con una balanza de presión. Se discute la ecuación propuesta por la guía DKD-R 6-1 para la estimación de incertidumbre, según plataforma GUM y Monte Carlo, proponiendo un modelo alternativo. Se determinan las diferencias que existen en las incertidumbres resultantes obtenidas según estos métodos y en qué forma los distintos componentes del modelo influyen en estas diferencias, según la magnitud de su incertidumbre estándar y la distribución de probabilidad (DDP) asociada que se le asigna.

El desarrollo se realiza según el método GUM utilizando la metodología analítica. Adicionalmente se repite el ejercicio en forma comparativa utilizando cuatro diferentes herramientas de cálculo. Dos de estas herramientas están basadas en aproximaciones numéricas del método clásico: software GUM Workbench $\odot$ y método numérico de Kragten. Las otras dos son consistentes con el suplemento 1 de la Guía de la Expresión de la Incertidumbre de Medida, según el método de Monte Carlo (MCM), en un caso el lenguaje R y por otra parte MCM Alchimia, aplicación desarrollada por uno de los autores.

Palabras clave: Metrología, presión, balanza de presión, incertidumbre, Monte Carlo, GUM, GUM Workbench, R, MCM Alchimia, Kragten, DKD-R 6-1.

Abstract

This paper focuses a study of the contributions of uncertainty and expanded uncertainty that take place in the calibration of a digital pressure gauge by direct comparison with a pressure balance. The work is performed based on the GUM method, using an analytical approach. The equation given by the DKD-R 6-1 guideline for uncertainty analysis is discussed proposing an alternative model. It is aimed to determine the differences in the resulting uncertainty, according to the different platforms used, and how the standard uncertainty and assigned probability function (FDP) associated to different magnitudes of the model impact these differences.

The development will be performed according to the GUM framework using the analytical methodology. In addition to this, the exercise is repeated in a comparative basis, using four calculation tools. Two of them are based on numerical approaches of the classical method, GUM Workbench software $\odot$ and Kragten method, and the rest are based on the Supplement 1 to the Guide to the Expression of Uncertainty of Measurement, in one case by mean of the R language and the second one using MCM Alchimia, application developed by one of the authors. Keywords: Metrology, Pressure, Pressure Balance, Uncertainty, Monte Carlo, Software, GUM, GUM Workbench, R, MCM Alchimia, Kragten, DKD-R 6-1.
\end{abstract}

\section{Introducción}

A partir del año 2008, el Laboratorio Tecnológico del Uruguay (LATU) ha realizado una fuerte inversión en patrones fundamentales de alta precisión para el laboratorio de presión, contando a la fecha con cinco ensambles Pistón-Cilindro de incertidumbres menores a $30 \mathrm{ppm}$, los cuales cubren un intervalo entre 0 y $70 \mathrm{MPa}$. En este marco el departamento de metrología científica del LATU comenzó a brindar apoyo a los laboratorios secundarios de calibración, ya sea dando trazabilidad a sus patrones de presión, así como participando en asesoramientos y organizando ensayos de aptitud con ellos. Las medidas sobre las cuales se realiza este estudio corresponden a las realizadas para la obtención de los valores de referencia en el ensayo de aptitud de la magnitud presión, realizado entre mayo y diciembre de 2013. Se estudian aquí los distintos aspectos y parámetros de entrada que involucran la estimación de incertidumbre según el método clásico (GUM), confrontándolos con resultados 
obtenidos a través de métodos estocásticos (MCM) y evaluando posibles explicaciones a las diferencias obtenidas.

Calibración de medidores digitales con balanza de presión. A medida que comienzan a estar disponibles en plaza balanzas de presión industriales de precios más accesibles, es de esperar que en los próximos años los laboratorios secundarios en Uruguay comiencen a utilizar estos equipos como patrones de referencia, dejando los instrumentos digitales de menor precisión y estabilidad como patrones de trabajo. Si bien la confiabilidad de los patrones de tipo fundamental es mayor a la de los equipos digitales, existen otras consideraciones que se deben tener en cuenta al realizar calibraciones con estos patrones, exigiendo en general una mayor competencia de los técnicos así como un mayor conocimiento de las características de su equipo específico. Dentro de las particularidades que tienen las calibraciones de este tipo es de destacar la diversidad de magnitudes que influyen en el proceso de comparación. Esto es debido a que al ser la presión una magnitud derivada la calibración de medidores se realiza en forma de comparación indirecta, obteniéndose la presión generada por el patrón de magnitudes tales como la masa aplicada en el pistón, densidad del aire circundante, área efectiva del ensamble pistón-cilindro, temperatura del mismo, gravedad local, etcétera, y no de un valor único de presión leído en el instrumento, corregido según su curva de calibración e incertidumbre asociada. En la medida que tanto los patrones utilizados como los calibrandos aumentan su precisión, muchas de estas magnitudes del modelo pueden llegar a alternar su significancia en la incertidumbre de calibración resultante. Esto no solo se observa para diferentes calibraciones, sino incluso a lo largo de una misma calibración; una magnitud de entrada puede pasar de ser un contribuyente menor a ser la controlante del proceso según la fracción del intervalo que se esté midiendo.

El propósito de este trabajo es evaluar estas influencias en una calibración específica y, al mismo tiempo, estudiar en qué medida las variantes inherentes a los procesos de cálculo pueden llegar a impactar en estos valores.

\section{Materiales y Métodos}

\section{Herramientas}

Existen numerosas herramientas informáticas en la actualidad para la estimación de incertidumbres de calibración, la mayoría de las cuales están basadas en la ley de propagación de incertidumbre. No obstante, a partir de la aprobación del suplemento 1 de la Guía para la Expresión de la Incertidumbre de Medida (JCGM, 2008a) también es posible estimar la incertidumbre del mesurando por medio de simulaciones de Monte Carlo. En este trabajo se utilizan las cuatro herramientas de cálculo que se detallan a continuación:

GumWorkbench. Este software comercial está ampliamente difundido y es consistente con los lineamientos de la guía JCGM 100:2008 (JCGM, 2008a). Como información de salida, esta aplicación permite obtener un presupuesto de incertidumbre con las incertidumbres estándar de los contribuyentes, la estimación de los grados de libertad efectivos del proceso y los resultados e incertidumbre expandida del mesurando.

Método de Kragten (Kragten, 1995). Este método sugiere una aproximación numérica a la obtención de los coeficien- tes de sensibilidad de los contribuyentes de incertidumbre, evitando por este medio el cálculo de las derivadas parciales del modelo y utilizando el potencial de las planillas de cálculo para realizar el proceso en forma estructurada. En este caso se sostiene que si asumimos que las incertidumbres típicas de las magnitudes de entrada son muy pequeñas con respecto al valor de esta, o que la función del mesurando $y=\mathrm{f}\left(x_{1}, x_{2}, \ldots\right.$ $x_{N}$ ) es lineal en $x_{i}$, es posible aproximar los coeficientes de sensibilidad según la expresión siguiente (Kragten, 1995):

$$
\frac{\delta_{f}}{\delta_{x}}=\frac{y\left(x_{i}+u\left(x_{i}\right)\right)-y\left(x_{i}\right)}{u\left(x_{i}\right)}
$$

Este método permite obtener los mismos elementos de salida que el GUM Workbench.

Lenguaje R. La estimación de incertidumbre del modelo según el método de Monte Carlo se diseñará primeramente en este lenguaje, ampliamente reconocido como herramienta para el cálculo estadístico. Por ser este un proceso estocástico no se obtendrá un resultado único, sino una población de resultados con una FDP asociada cuyos parámetros estadísticos permiten obtener un valor medio y un intervalo de confianza como resultados para el mesurando.

MCM Alchimia (Constantino, 2013). Esta aplicación también utiliza el método de Monte Carlo para la estimación de incertidumbres. Como información de salida también permite obtener las contribuciones de incertidumbre para los parámetros de entrada en el modelo. Estos valores los obtiene realizando simulaciones de estas magnitudes, manteniendo el resto de las variables fijas en su esperanza matemática.

\section{Materiales}

El patrón utilizado es una Balanza de Presión Neumática marca DHI modelo PG-7102 con un ensamble pistón-cilindro reentrante de relación de masa $200 \mathrm{kPa} / \mathrm{kg}$ modelo PC7100/7600-200. Como calibrando se utiliza un manómetro digital marca FLUKE modelo 700G07. El método utilizado está basado en DKD-R 6-1 (Deutscher Kalibrierdienst, 2003) y se realiza para 10 puntos de la escala de medida que cubre un intervalo de 0,34 a 3,40 $\mathrm{MPa}$.

\section{Modelo matemático de la calibración}

El modelo resultante en una calibración de este tipo podría subdividirse en tres etapas. En primer lugar, el cálculo de parámetros del modelo como la densidad del fluido $\left(\mathrm{N}_{2}\right)$, dependiente de la presión medida, o la densidad del aire, dependiente de las condiciones ambientales durante el ensayo. Luego es calculada la presión generada por el patrón, y en la última etapa estos valores son utilizados en la propia calibración del instrumento de muestra.

La presión generada en el caso de balanzas neumáticas puede expresarse según el siguiente modelo matemático, DKD-R 6-1 ec. 31 y 32 (Deutscher Kalibrierdienst, 2003):

$$
\begin{aligned}
P= & \frac{\sum M \cdot g l \cdot\left(1-\frac{\rho_{a}}{\rho_{m}}\right)}{A_{(0, t r)} \cdot\left[1+\alpha\left(t_{p}-t_{r}\right) \cdot\left(1+\lambda \cdot P_{N}\right)\right.} \\
& +\left[\left(\rho_{f}-\rho_{a}\right) \cdot g l \cdot \Delta h\right]
\end{aligned}
$$


Donde:

IM: Masa total en $\mathrm{kg}$.

$g l$ : Acel. de la gravedad local en $\mathrm{m} / \mathrm{s}^{2}$.

$\rho_{a}$ : Densidad del aire en $\mathrm{kg} / \mathrm{m}^{3}$.

$\rho_{m}$ : Densidad de las masas en $\mathrm{kg} / \mathrm{m}^{3}$.

$\rho_{f}$ : Densidad del fluido $\left(\mathrm{N}_{2}\right)$ en $\mathrm{kg} / \mathrm{m}^{3}$.

$A_{(0, t r}:$ Área efectiva del ensamble a presión cero

y t de referencia $\left(20^{\circ} \mathrm{C}\right) \mathrm{m}^{2}$.

$\alpha$ : Coef. de expansión térmica en ${ }^{\circ} \mathrm{C}^{-1}$

$T_{p}$ : Temperatura del pistón en ${ }^{\circ} \mathrm{C}$.

$T_{r}$ : Temperatura de referencia en ${ }^{\circ} \mathrm{C}$.

$\lambda$ : Coeficiente de deformación con $\mathrm{P}$ en $\mathrm{Pa}^{-1}$.

$P_{N}$ : Presión Nominal en Pa.

$\Delta h$ : Diferencia de nivel entre el patrón

y el calibrando en $\mathrm{m}$.

En esta ecuación debe hacerse notar que algunos parámetros surgen de cálculos independientes, por ejemplo, la densidad del aire $\left(\rho_{a}\right)$ responderá a la ecuación CIPM-2007 (Picard, et al., 2008):

$\rho=\frac{p \cdot M_{a}}{Z \cdot R \cdot T}\left[1-x_{v} \cdot\left(1-\frac{M_{v}}{M_{a}}\right)\right]$

Con:

$\rho$ : Densidad del aire en $\mathrm{kg} / \mathrm{m}^{3}$.

$p$ : Presión atmosférica en $\mathrm{Pa}$.

$M a$ : Masa molar de aire seco $=28,96546 \cdot 10^{-3} \mathrm{~kg} / \mathrm{mol}$.

$Z$ : Factor de compresibilidad adimensional.

$R$ : Constante molar de los gases.

$T$ : Temperatura en $\mathrm{K}$.

$x_{\mathrm{v}}$ : Fracción molar de vapor de agua adimensional.

$M_{\mathrm{v}}=$ Masa molar de vapor de agua $0,01801528 \mathrm{~kg} / \mathrm{Mol}$.

El valor de $\mathrm{x}_{\mathrm{v}}$ se determina a partir de:

$x_{v}=\frac{h}{100}\left(\alpha+\beta \cdot p+\gamma \cdot t^{2}\right) \cdot \frac{e^{\left(A T^{2}+B T+C+\frac{D}{T}\right)}}{P}$

Mientras que $Z$ está determinada por la fórmula:

$$
\begin{aligned}
Z= & 1 \frac{P}{T}\left[a_{0}+a_{1} t+a_{2} t^{2}+\left(b_{0}+b_{1} t\right) x_{v}\right. \\
& \left.+\left(c_{0}+c_{1} t\right) x_{v}^{2}\right]+\frac{p^{2}}{T^{2}}\left(d+e x_{v}^{2}\right)
\end{aligned}
$$

Con:

$t$ : Temperatura ambiente ${ }^{\circ} \mathrm{C}$.

$h:$ Humedad relativa.

$\alpha: 1,0062$.

$\beta: 3,14 \times 10^{-8} \mathrm{~Pa}^{-1}$.

$\gamma: 5,6 . \times 10^{-7} \mathrm{k}^{-2}$.

A : $1,2378847 \times 10^{-5} \mathrm{k}^{-2}$.

B : $-1,9121316 \times 10^{-2} \mathrm{k}^{-1}$

C : 33,93711047 .

D : $-6,3431645 \times 10^{-3} \mathrm{~K}$.

$\mathrm{a}_{\mathrm{o}}=1,58123 \times 10^{-6} \mathrm{KPa}^{-1}$.

$\mathrm{a}_{1}=-2,9331 \times 10^{-8} \mathrm{~Pa}^{-1}$.

$\mathrm{a}_{2}=1,1043 \times 10^{-10} \mathrm{~K}^{-1} \mathrm{~Pa}^{-1}$.

$\mathrm{b}_{0}=5.707 \times 10^{-6} \mathrm{KPa}^{-1}$.

$\mathrm{b}_{1}=-2,051 \times 10^{-8} \mathrm{~Pa}^{-1}$.

$\mathrm{c}_{0}=1,9898 \times 10^{-4} \mathrm{KPa}^{-1}$.

$c_{1}=-2,376 \times 10^{-6}$.

$\mathrm{d}=1,83 \times 10^{-11} \mathrm{k}^{2} \mathrm{~Pa}^{-2}$.

$\mathrm{e}=-0,765 \times 10^{-8} \mathrm{~K}^{2} \mathrm{~Pa}^{-2}$.

Puede verse que la densidad del aire dependerá de las condiciones de humedad y temperatura ambiente, además de la presión atmosférica. En este trabajo se calcula el intervalo de valores de $h, t$ y $P$ durante la calibración. A partir de esta ecuación se obtiene un valor único de $\rho_{\mathrm{a}}$ al que se le asigna la incertidumbre calculada según método analítico. Este valor y su incertidumbre asociada son válidos tanto para el método GUM como para MMC (Constantino, 2013).

En el caso de la temperatura del ensamble pistón-cilindro, su valor será la media de toda la calibración con una incertidumbre indicada por el informe de calibración del sensor de temperatura, a la que se adicionará una variable de variación de temperatura de valor cero $\left(\Delta t_{p}\right)$ cuya incertidumbre será representada por medio de una FDP uniforme con intervalo en sus cotas máxima y mínima.

Otro parámetro variable que no puede obtenerse directamente es la densidad del fluido, en este caso Nitrógeno. Debe tenerse en cuenta que esta densidad variará significativamente en cada punto medido del intervalo de lectura, dependiente de la presión $(p)$ y temperatura $(T)$, y responderá a la expresión:

$\rho_{f(T, P)}=\rho_{f(T 0, p 0)} \cdot \frac{p}{p 0} \cdot \frac{T 0}{T}$

Donde:

$\rho_{f(T, P)}$ : Densidad del Nitrógeno a temperatura T y presión $\mathrm{p}$. $\rho_{f(T 0, p))}: 1,25046 \mathrm{~kg} \cdot \mathrm{m}^{-3}$.

$\rho 0: 1013,25 \mathrm{hPa}$.

T0: $273,15 \mathrm{~K}$.

Para este parámetro se asignará una incertidumbre del $5 \%$ de su valor, según una DDP uniforme.

Otro parámetro que variará es el set de masas utilizado. La densidad de las mismas es $8000 \mathrm{~kg} / \mathrm{m}^{3}$, ya que tanto las pesas como el pistón y la campana de soporte están calibrados en masa convencional.

Finalmente, debe tenerse en cuenta la ecuación propia de la calibración del instrumento digital. Esta puede expresarse para la corrección media ascendente/descendente según la guía DKD-R 6-1 ec. 9 y 10 (Deutscher Kalibrierdienst, 2003):

$$
\begin{aligned}
C_{i}= & \Delta p_{i}=p p_{i}-p l_{i}+\left(\delta_{p . \text { desv.cero }}\right. \\
& \left.+\delta_{\text {p.repetibilidad }}+\delta_{\text {p.histéresis }}\right)_{i}
\end{aligned}
$$

Donde $p p_{i}$ corresponde a la presión generada por el patrón, $p l_{i}$ al promedio de lecturas del calibrando en el intervalo $i, \delta_{\text {p.desv.cero }}, \delta_{\text {p.repetibilidad }}$ y $\delta_{\text {p.histéresis }}$ corresponden a las contribuciones de incertidumbre para la desviación de cero, la repetibilidad y la histéresis, respectivamente.

La ecuación resultante para la corrección media en cada punto $i$ del intervalo calibrado es entonces:

$$
\begin{aligned}
C_{i}= & \frac{\sum M_{i} \cdot g l \cdot\left(1-\frac{\rho_{a}}{\rho_{m}}\right)}{A_{(0, t r) \cdot} \cdot\left\{1+\alpha\left[\left(t_{p}+\Delta t_{p}\right)-t_{r}\right] \cdot\left(1+\lambda \cdot P_{N i}\right)\right\}} \\
& +\left\{\left[\left(1,25046 \cdot \frac{\left(P_{N i}+1 \times 10^{5}\right)}{101325} \cdot \frac{273,15}{273,15+t_{p}}\right)-\rho_{a}\right]\right. \\
& \cdot g l . \Delta h\}-p l_{i} \\
& +\left(\delta_{\text {p.desv.cero }}+\delta_{\text {p.repetibilidad }}+\delta_{\text {p.histéresis }}\right)_{i}
\end{aligned}
$$

Para simplificar el cálculo de los coeficientes de sensibilidad en el método analítico el cálculo se realiza en forma secuencial según la ecuación: 
$C_{i}=\frac{F_{i}}{A_{i}}+C n r-p l_{i}+\left(\delta_{p . \text { desv.cero }}+\delta_{\text {p.repetibilidad }}\right.$

$$
\left.+\delta_{\text {p.histéresis }}\right)_{i}
$$

Donde: F representa la fuerza aplicada, A el área efectiva del ensamble pistón-cilindro y Cnr la corrección por nivel de referencia.

$$
\begin{aligned}
& F=\sum M \cdot g l \cdot\left(1-\frac{\rho_{a}}{\rho_{m}}\right) \\
& A=A_{(0, t r) \cdot} \cdot\left\{1+\alpha\left[\left(t_{p}+\Delta t_{p}\right)-t_{r}\right] \cdot\left(1+\lambda \cdot P_{N i}\right)\right\} \\
& C n r=\left\{\left[\left(1,25046 \cdot \frac{P_{N i}}{1013,25} \cdot \frac{273,15}{273,15+\left(t_{p}+\Delta t_{p}\right)}\right)\right.\right. \\
& \left.\left.\quad-\rho_{a}\right] \cdot g l \cdot \Delta h\right\}
\end{aligned}
$$

\section{Resultados}

En el ensayo realizado las componentes de desviación de cero y de repetibilidad, calculada como el desvío estándar de 10 medidas, arrojaron un valor nulo. Por su parte, solamente los puntos correspondientes a $0,68 \mathrm{MPa}, 1,36 \mathrm{MPa}$ y 2,38 $\mathrm{MPa}$ presentaron histéresis no nulas, de valor $100 \mathrm{~Pa}$ (una división) en todos los casos. Estos puntos se marcan con fondo oscuro en las tablas para facilitar la visualización de las conclusiones.

La Tabla 1 resume los resultados e incertidumbre obtenidos para cada intervalo, según los métodos de cálculo utilizados. Para poder realizar una evaluación comparativa entre los métodos de cálculo según el concepto de tolerancia numérica, se expresan las incertidumbres obtenidas con un número mayor de cifras significativas al indicado según GUM. Como criterio de comparación se utiliza el concepto de tolerancia numérica $\delta$ de la incertidumbre (JCGM, 2008b, pp. 31-35).

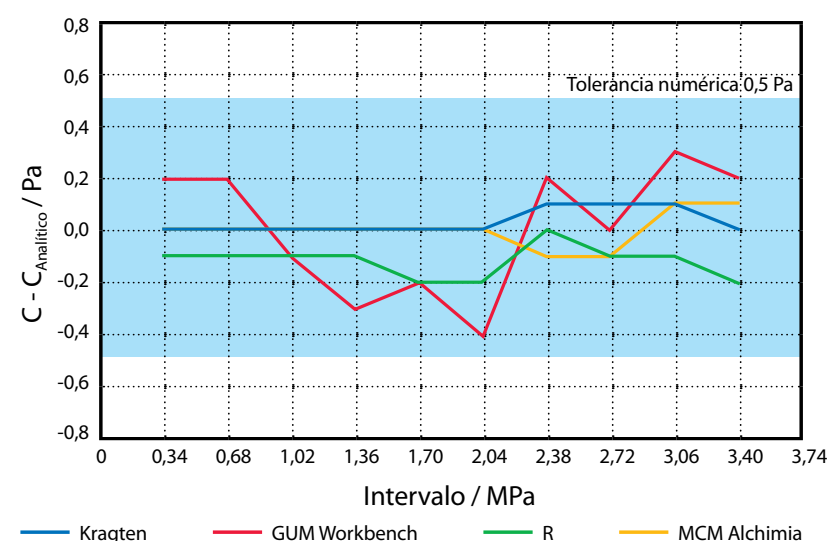

Gráfico 1. Resultados del mesurando según los cinco métodos de cálculo y tolerancia numérica de $U$.

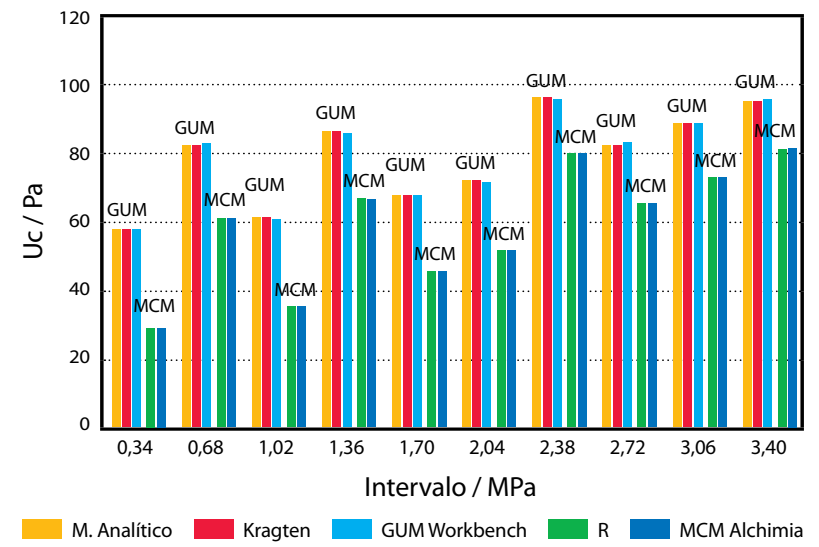

Gráfico 2. Diagrama comparativo de incertidumbre expandida, métodos GUM y MCM.

\begin{tabular}{|c|c|c|c|c|c|c|}
\cline { 2 - 7 } & \multicolumn{4}{c|}{ Resultados del mesurando-Corrección/Pa } & \multicolumn{2}{c|}{ Criterio } \\
\cline { 2 - 7 } & GUM & Aproximación numérica (GUM) & \multicolumn{2}{c|}{ Monte Carlo } & Tol. numérica \\
\hline \multirow{2}{*}{ Nominal/MPa } & Analítico & Kragten & $\begin{array}{c}\text { GUM } \\
\text { Workbench }\end{array}$ & R & $\begin{array}{c}\text { MCM } \\
\text { Alchimia }\end{array}$ & $\delta /$ Pa \\
\hline 0,34 & $-2,2$ & $-2,2$ & -2 & $-2,3$ & $-2,2$ & 0,5 \\
0,68 & 33,8 & 33,8 & 34 & 33,7 & 33,8 & 0,5 \\
1,02 & 26,1 & 26,1 & 26 & 26,0 & 26,1 & 0,5 \\
1,36 & 137,3 & 137,3 & 137 & 137,2 & 137,3 & 0,5 \\
1,70 & 194,2 & 194,2 & 194 & 194,0 & 194,2 & 0,5 \\
2,04 & 320,4 & 320,4 & 320 & 320,2 & 320,4 & 0,5 \\
2,38 & 337,8 & 337,9 & 338 & 337,8 & 337,7 & 0,5 \\
2,72 & 403,0 & 403,1 & 403 & 402,9 & 402,9 & 0,5 \\
3,06 & 537,7 & 537,8 & 538 & 537,6 & 537,8 & 0,5 \\
3,40 & 375,8 & 375,8 & 376 & 375,6 & 375,9 & 0,5 \\
\hline
\end{tabular}

Tabla 1. Resultados del mesurando según los cinco métodos de cálculo. 


\begin{tabular}{|c|c|c|c|c|c|}
\cline { 2 - 6 } \multicolumn{1}{c|}{} & \multicolumn{5}{c|}{ Evaluación de incertidumbre expandida- $\mathrm{U}_{\mathrm{C}} / \mathrm{Pa}$} \\
\cline { 2 - 6 } & GUM & Aproximación numérica (GUM) & \multicolumn{2}{c|}{ Monte Carlo } \\
\hline Nominal/MPa & M. Analítico & Kragten & GUM Workbench & $\mathrm{R}$ & MCM Alchimia \\
\hline 0,34 & 58,2 & 58,2 & 58 & 29,3 & 29,3 \\
0,68 & 82,8 & 82,8 & 83 & 61,5 & 61,3 \\
1,02 & 61,5 & 61,5 & 61 & 35,6 & 35,5 \\
1,36 & 86,4 & 86,4 & 86 & 66,9 & 66,7 \\
1,70 & 67,8 & 67,8 & 68 & 45,7 & 45,8 \\
2,04 & 72,1 & 72,1 & 72 & 51,8 & 51,8 \\
2,38 & 96,2 & 96,2 & 96 & 80,2 & 80,5 \\
2,72 & 82,6 & 82,6 & 83 & 65,5 & 65,7 \\
3,06 & 88,7 & 88,7 & 89 & 73,4 & 73,3 \\
3,40 & 95,5 & 95,5 & 96 & 81,4 & 81,5 \\
\hline
\end{tabular}

Tabla 2. Incertidumbre expandida del mesurando según los cinco métodos de cálculo.

\section{Discusión}

En referencia a los resultados de calibración, el Gráfico 1 muestra que no existen diferencias significativas entre ninguno de los enfoques o herramientas de cálculo utilizados en lo que refiere al resultado del mesurando. Es pertinente notar que la mayor variación corresponde al método GUM Workbench. La magnitud de estas diferencias puede deberse a que en este software no pueden obtenerse los resultados con más cifras significativas de las que determina la expresión de incertidumbre de acuerdo a GUM (JCGM, 2008a), lo que aporta una diferencia mayor con respecto al valor calculado analíticamente, debido al redondeo.

Por su parte, la Tabla 2 y el Gráfico 2 expresan que no existen diferencias significativas entre los valores obtenidos utilizando herramientas basadas en la plataforma GUM, como tampoco existen entre aquellas herramientas que permiten realizar el cálculo por medio de simulaciones de Monte Carlo (R y MCM Alchimia). Sin embargo, existe un importante sesgo entre las incertidumbres expandidas obtenidas por medio de estos dos métodos estadísticos, GUM y MCM.

La guía JCGM101:2008 (JCGM, 2008b, p. 34) indica que en caso de diferencias entre GUM y MCM mayores que la tolerancia numérica $\delta$, el valor de MCM debe ser considerado correcto, no obstante diferencias de esta magnitud no pueden ser explicadas por un cambio en el enfoque de cálculo, sino que deben hacerse consideraciones al modelo matemático propuesto en cada caso que permitan justificar esas diferencias. Se evalúan posibles contribuyentes de incertidumbre según el método analítico GUM y comparan con los valores obtenidos para el MCM mediante el lenguaje R.

La Tabla 3 muestra que no existen diferencias significativas entre las incertidumbres obtenidas para la presión generada según GUM y MCM. Como se planteó anteriormente, la repetibilidad y el desvío de cero arrojaron incertidumbre nula. Por otra parte, la histéresis solo influye en tres de los puntos, por lo que el sesgo no puede ser explicado por los valores de incertidumbres de los contribuyentes individuales.

\section{Incertidumbre en la presión generada por el patrón}

\begin{tabular}{|c|c|c|}
\hline & \multicolumn{2}{|c|}{$\mathrm{U}_{\text {P.Patrón }} / \mathrm{Pa}$} \\
\hline Nominal/MPa & GUM & MCM \\
\hline 0,34 & 6,98 & 6,96 \\
0,68 & 14,0 & 13,9 \\
1,02 & 21,1 & 21,0 \\
1,36 & 28,2 & 28,2 \\
1,70 & 35,6 & 35,5 \\
2,04 & 43,2 & 43,0 \\
2,38 & 50,9 & 50,8 \\
2,72 & 59,0 & 58,9 \\
3,06 & 67,4 & 67,3 \\
3,40 & 76,1 & 75,9 \\
\hline
\end{tabular}

Tabla 3. Incertidumbre expandida de presión del patrón según GUM y MCM.

\section{Cálculo de $\mathrm{U}_{\mathrm{pl}}$ según DKD-R 6-1}

La guía DKD-R 6-1:2003 (Deutscher Kalibrierdienst, 2003) propone utilizar el valor promedio de las lecturas con una FDP uniforme de intervalo igual al valor de la división del calibrando. Por ejemplo, en el caso de la ec. 1 de esta guía se indica que:

$$
U_{\text {up } / \text { down }}=k \cdot \sqrt{\begin{array}{l}
u_{\text {standard }}^{2}+u_{\text {resolution }}^{2} \\
+u_{\text {zero-deviation }}^{2}+u_{\text {repeatibility }}^{2}
\end{array}}
$$


Sin embargo, este modelo de estimación no toma en cuenta que el valor de lectura del calibrando (pl) está compuesto por un promedio de dos distribuciones uniformes, lo que resulta en una distribución triangular de probabilidad, con intervalo igual a la resolución del calibrando. Como en el ensayo se realiza en base a dos corridas, también debe evaluarse si el valor de esta magnitud es obtenido como el promedio ascendente/ descendente en una corrida o si se promedian además ambas corridas. En este último caso tampoco es correcto utilizar esta distribución triangular, ya que el valor de $\mathrm{P}$ es obtenido de promediar nuevamente dos magnitudes con FDP triangular o las cuatro lecturas con distribución uniforme.

El Gráfico 3 compara la incertidumbre estándar en la lectura del calibrando (pl) aplicando la ley de propagación de incertidumbres a las cuatro opciones anteriores.

$\mathrm{u}_{\mathrm{pl}}$ según forma de cálculo

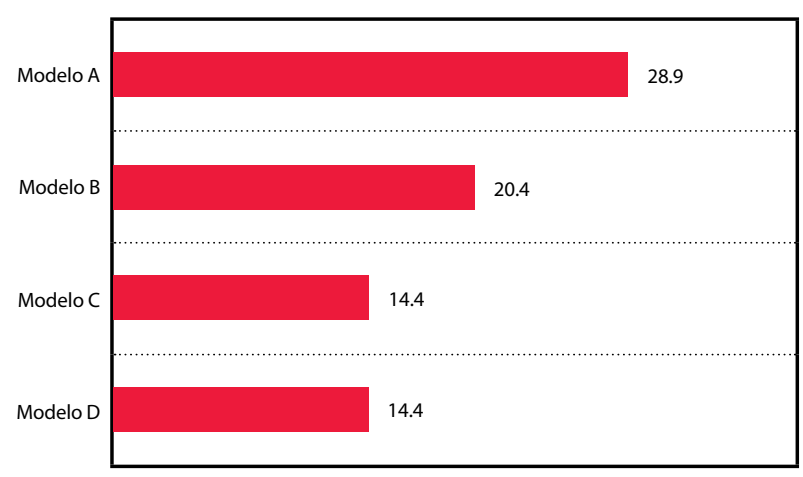

Gráfico 3. Incertidumbre estándar de pl según 4 modelos de cálculo.

Donde:

Modelo A: Cálculo según DKD-R 6-1.

Modelo B: $\mathrm{pl}=\left(\mathrm{pl}_{\text {ascenso }}+\mathrm{pl}_{\text {descenso }}\right) / 2$.

Modelo C: $\mathrm{pl}=\left(\mathrm{pl}_{\mathrm{AD} \text {,corridal }}+\mathrm{pl}_{\mathrm{AD} \text {,corrida2 }}\right) / 2$.

Modelo D: $\mathrm{pl}=\left(\mathrm{pl}_{1}+\mathrm{pl}_{2}+\mathrm{pl}_{3}+\mathrm{pl}_{4}\right) / 4$.

En este caso $\mathrm{pl}_{\mathrm{AD}}$ corresponde al valor promedio ascenso/ descenso a cuya incertidumbre se asigna distribución triangular resultante del promedio, mientras que $\mathrm{pl}_{\mathrm{n}}$ corresponde a lecturas individuales con FDP uniforme. Es pertinente notar que el resultado en los casos $\mathrm{C}$ y D son iguales entre sí pero diferentes a los obtenidos según modelos A y B, ya que estos últimos incluyen dos lecturas adicionales.

\section{Cálculo de $U_{p p}$ según DKD-R 6-1}

La misma consideración anterior puede hacerse en el caso de la presión generada por el patrón, que es tomada cuatro veces y de ellas se obtendrá un promedio. Sin embargo, en este ensayo en el que el patrón es una balanza de presión las incertidumbres controlantes del modelo son las correspondientes a la calibración de las masas y de las dimensiones y coef. de deformación $\lambda$ del ensamble pistón/cilindro. Es razonable que estas magnitudes de contribución tipo B se consideren correlacionadas con coeficiente de correlación de 1 en las cuatro medidas, por lo que en este caso puede suponerse una sola medida del patrón y es aceptable suponer el modelo propuesto por DKD-R 6-1 (Deutscher Kalibrierdienst, 2003) para este parámetro.

\section{Cálculo de $\mathrm{U}_{\Delta \mathrm{P}}$ según $\mathrm{MCM}$}

El modelo MCM, libre de consideraciones deterministas, implica considerar todas las operaciones necesarias para arribar al resultado, las cuales impactan en el resultado de incertidumbre, tanto en su valor como en su distribución de probabilidad. En el modelo matemático propuesto para MCM se promediaron los valores de corrección =pp-pl en las cuatro lecturas:

$\Delta P=\frac{\left(p p_{1}-p l_{1}\right)+\left(p p_{2}-p l_{2}\right)+\left(p p_{3}-p l_{3}\right)+\left(p p_{4}-p l_{4}\right)}{4}$

Donde $\mathrm{pp}_{\mathrm{i}}$ corresponde a la presión generada por el patrón en la corrida i y $\mathrm{pl}_{\mathrm{i}}$ al valor correspondiente en la lectura del calibrando. Este modelo con el cual se realizaron los cálculos primarios no tiene en cuenta, no obstante, la correlación existente entre las lecturas del patrón mencionada antes.

\section{Cálculo comparativo con el modelo matemático de DKD-R 6-1 corregido}

La Tabla 4 expone la incertidumbre expandida para las correcciones aplicando los métodos GUM (analítico) y MCM (software R) al mismo modelo corregido según las consideraciones anteriores:

$$
\begin{aligned}
C_{i}= & p p_{i}-\left(\frac{\left(p_{1 . i}+p_{2, i}+p_{3, i}+p_{4, i}\right)}{4}\right)_{i}+\left(\delta_{p, \text { desv.cero }}\right. \\
& \left.+\delta_{\text {p.repetibilidad }}+\delta_{p . \text { histéresis }}\right)_{i}
\end{aligned}
$$

\begin{tabular}{|c|c|c|c|c|}
\hline & \multicolumn{2}{|c|}{$\mathrm{U}_{\mathrm{C}} / \mathrm{Pa}$} & \multicolumn{2}{c|}{} \\
\hline Nominal/MPa & GUM & MCM & $\Sigma$ & $k$ calculado \\
\hline 0,34 & 29,7 & 29,3 & 14,9 & 1,97 \\
0,68 & 66,0 & 61,5 & 33,1 & 1,85 \\
1,02 & 35,7 & 35,6 & 17,8 & 2,00 \\
1,36 & 70,5 & 66,9 & 35,2 & 1,90 \\
1,70 & 45,8 & 45,7 & 22,9 & 2,00 \\
2,04 & 51,9 & 51,8 & 26,0 & 1,99 \\
2,38 & 82,2 & 80,1 & 41,0 & 1,95 \\
2,72 & 65,7 & 65,6 & 32,8 & 2,00 \\
3,06 & 73,3 & 73,5 & 36,7 & 2,00 \\
3,40 & 81,4 & 81,4 & 40,7 & 2,00 \\
\hline
\end{tabular}

Tabla 4. Incertidumbre expandida del modelo matemático corregido.

Puede notarse primeramente a partir de esta Tabla 4 (en segunda y tercera columna) que los resultados en MCM, contemplando las magnitudes correlacionadas, son idénticos a los calculados sin tener en cuenta esta consideración (Tabla 2 , columna «R»). Una segunda observación es que la mayoría de los puntos arrojan resultados idénticos entre GUM y MCM, excepto en tres de ellos que son precisamente aquellos que presentan histéresis no nula. 
El Gráfico 4 presenta histogramas de frecuencias de la simulación, aportados por el software R, para dos puntos consecutivos, uno de ellos con histéresis no nula (Punto 0,68 $\mathrm{MPa}$ ) y el otro (Punto 1,02 MPa) sin aportar incertidumbre por histéresis.
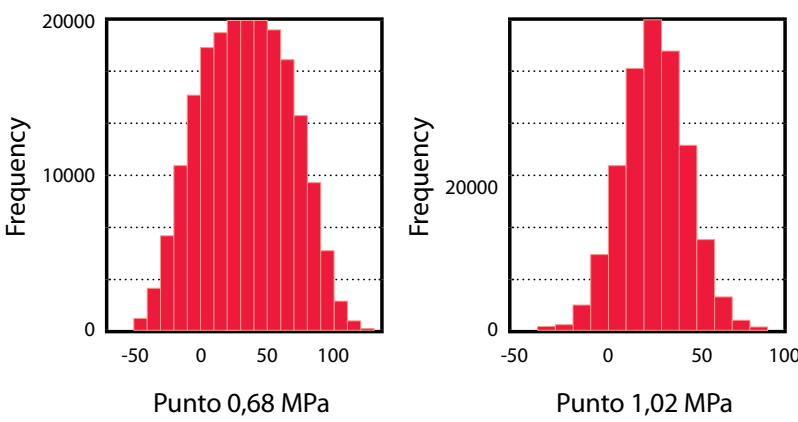

Gráfico 4. Histogramas comparativos para puntos con y sin histéresis.

En el primer caso puede verse que la histéresis (con FDP uniforme) «uniformiza» en buena medida la función distribución resultante del modelo. Al perder su forma gaussiana, la plataforma GUM se aleja de los resultados de MCM y por tanto deja de tener validez como estimador de incertidumbre para el modelo. El menor valor de $U$ en estos casos es debido a que para un nivel de confianza del $95,45 \%$ el factor de cobertura que se obtiene de GUM para un resultado con FDP Normal es de 2 , en tanto si la función distribución fuera perfectamente uniforme, el factor de cobertura para un nivel de confianza de $95,45 \%$ es cercano al 1,7 (JCGM, 2008a, p. 70). La cuarta y quinta columna de la Tabla 4 muestran el desvío estándar $(\sigma)$ del vector resultante por MCM y su $k$ calculado como $U_{C} / \sigma$ a partir de esta colección de resultados. Se observa que en los puntos con histéresis no nula el $k$ calculado es de menor valor que el supuesto para una distribución normal, mientras que el resto pueden asumirse normales, como supone la plataforma GUM de acuerdo al teorema del límite central.

\section{Conclusiones}

Modelo DKD-R 6-1 (Deutscher Kalibrierdienst, 2003). El modelo de estimación de incertidumbre propuesto por esta guía no resultó validado por los valores obtenidos por MCM. Este cálculo arroja valores de $U$ sobreestimados, ya que no tienen en cuenta la obtención del valor de lectura del calibrando a partir de un promedio de lecturas independientes. Estos resultados erróneos pueden resolverse realizando el cálculo a partir del modelo corregido propuesto en este artículo (Tabla 4).

GUM vs. MCM. Se observa que el método GUM aplicado al modelo corregido puede considerarse válido en tanto una magnitud de entrada con FDP uniforme no sea la magnitud controlante del proceso. En este último caso, el factor de cobertura $k$ tomará valores menores a 2 para un nivel de confianza de $95,45 \%$, por lo que el método GUM en estos puntos no es aplicable. Debe tenerse en cuenta que en la calibración de instrumentos de baja precisión estas magnitudes uniformes pueden ser controlantes en todo el intervalo de lectura, por lo que el método GUM podría no ser válido (JCGM, 2008b, p. 34) en ningún punto a lo largo del intervalo de lectura del instrumento.

Magnitudes correlacionadas. No se encontraron diferencias entre los valores calculados teniendo en cuenta la correlación existente para valores del patrón y los resultados obtenidos considerando todas magnitudes independientes.

Herramientas de cálculo. No se encontraron diferencias entre los resultados de calibración obtenidos por las diferentes herramientas de cálculo aplicadas, obteniéndose resultados idénticos al método analítico a partir de las cuatro herramientas alternativas. Respecto al valor de incertidumbre expandida, se obtuvieron valores equivalentes al método analítico según las herramientas basadas en la ley de propagación de incertidumbres (métodos Kragten y GUM Workbench), así como resultados equivalentes entre sí para las dos herramientas basadas en el método de Monte Carlo (software R y MCM Alchimia).

\section{Reconocimientos}

Se agradece al personal del departamento de Metrología Química del LATU, que organizó el ensayo de aptitud que permitió la realización de este estudio.

\section{Referencias}

Constantino, Pablo, 2013. Aspectos computacionales en la estimación de incertidumbres de ensayo por el Método de Monte Carlo. En: INNOTEC, (8), pp.3-22.

Deutscher Kalibrierdienst, 2003. Guideline DKD-R 6-1. Calibración of pressure gauge [En línea]. Braunschweig: DKD. [Consulta 10 de abril de 2014]. Disponible en: http:// www.dkd.eu/dokumente/Richtlinien/dkd_r_6_1_e.pdf JCGM, 2008a. JCGM 100. Evaluation of measurement data - Guide to the expression of uncertainty in measurement. [En línea]. Sèvres: JCGM. [Consulta 2 de mayo de 2013] Disponible en: http://www.bipm.org/utils/common/documents/jcgm/ JCGM_100_2008_E.pdf

JCGM, 2008b. JCGM 101. Evaluación of measurement dataSupplement 1 to the "Guide to the expression of uncertainty in measurement" - Propagation of distributions using a Monte Carlo method [En línea]. Sèvres: JCGM. [Consulta 2 de mayo de 2013] Disponible en: http://www.bipm.org/ utils/common/documents/jcgm/JCGM_101_2008_E.pdf

Kragten, J., 1995. A standard scheme for calculating numerically standard deviation and confidence intervals. En: Chemometrics and Intelligent Laboratory Systems, (28), pp.89-97.

Picard, A., Davis, R.S., Glaser, M., Fujii, K., 2008. Revised formula for the density of moist air (CIPM-2007). En: Metrologia, (45), pp.149-155. 